\title{
Дискуссии
}

УДК 541.183 .12

\section{Продолжение обсуждения статьи В.А. Шапошника «Электростатическая теория селективности ионообменников»}

\author{
(C) 2021 Хамизов P.X. \\ Институт геохимии и аналитической химии (ГЕОХИ) РАН, Москва \\ Поступила в редакцию 06.06.2021 г.
}

DOI: $10.17308 /$ sorpchrom.2021.21/3645

Дискуссионная статья Р.Х. Хамизова посвящена обсуждению материалов, представленных профессором В.А. Шапошником в журнале «Сорбционные и хроматографические процессы» (2021, Т. 21, № 1) В статье Р.Х. Хамизова и А.М.Долгоносова «К вопросу о статье В.А. Шапошника «Электростатическая теория селективных ионообменников» рассмотрены подходы к этой проблеме, в том числе, в модели Дж. Поли для оценки констант равновесия ионного обмена.

Более корректной моделью селективности представляется модель Дж. Эйзенмана, хотя она выступает только в роли качественной характеристики. Привлекательность модели Эйзенмана вытекает из того, что она неподгоночная. Эйзенман использовал в своих расчетах табличные значения кристаллохимических радиусов, считая, что между фиксированным ионом и противоионом нет молекул воды.

Критические замечания в адрес В.А. Шапошника в своем подходе к теории селективности сделаны в связи с тем, что, развивая какую-то гипотезу, необходимо пользоваться не только первыми шагами предшественников, но и последующими (или наиболее признаваемыми) достижениями. В этом плане выражено несогласие с позицией В.А. Шапошника в том, что он отказывается от энергии дегидратации. Неучет энергий дегидратации при рассмотрении селективности ионообменников приводит к очень большим ошибкам. Следует отметить, что модель качественную, основанную на энергии кулоновского взаимодействия, до количественных оценок успешно довел Д. Райхенберг, введя в соответствующие соотношения термы для энергий близкодействующих взаимодействий, включая дисперсионные.

Автор данной статьи считает, что поправлять расчеты с помощью дробных зарядов в точечной модели Кулона (где не предусмотрена делокализация) нецелесообразно. Рассматривая применимость уравнения Пуассона к ионообменникам, автор этой статьи рекомендует ознакомиться с его работами (в соавторстве с Н.А. Тихоновым), где были решены очень сложные задачи для сильноосновных анионообменников и гидрогелей на основе графена.

Ключевые слова: ионный обмен, модели селективности, кулоновское взаимодействие, энергия гидратации, водородная связь.

Статья В.А. Шапошника, опубликованная в 2020 году в 1-м номере журнала «Сорбционные и хроматографические процессы» [1], вызвала дискуссию, так как посвящена одной из наиболее важных и фундаментальных проблем в ионном обмене - проблеме селективности. В 5-м номере Журнала были опубликованы наши с А.М. Долгоносовым ком- ментарии к этой статье [2], а во 2-м номере этого года появилась публикация с ответами и разъяснениями от Владимира Алексеевича [3]. После прочтения этих ответов нам показалось, что не все наши комментарии в [2] были поняты (или понятны), что еще раз возвращает нас к обсуждению этой интересной темы. При этом мы, конечно, обойдемся без реак- 
ции на справедливые слова, высказанные в [3] прямо или косвенно о том, какими должны быть авторы, а также постараемся опустить очевидные материалы учебного характера. Повторим только еще раз тезис из [2], что кроме подхода к расчету термодинамических констант равновесия ионного обмена в виде формулы (2), приведенной в [3] с пределами интегрирования по Дж. Гейнсу и Г. Томасу, есть еще подход, в соответствии с которым в качестве стандартных условий для ионообменников принимаются условия для бесконечно разбавленного раствора электролита, термодинамическая константа принимается равной единице, а селективность ионного обмена определяется отношением коэффициентов активностей конкурирующих ионов в фазе ионита.

1. Почему мы считаем (с позиций сегодняшних знаний) не вполне корректной модель Дж. Поли, изложенную в его очень актуальной и приоритетной работе [4]? При переборе различных известных (из литературы того времени) значений радиусов гидратированных ионов автор выбрал параметры, соответствующие радиусам наибольшего сближения ионов в теории Дебая-Хюккеля. Именно их подстановка в уравнение модели позволяла в наибольшей степени приблизиться к экспериментальным значениям констант равновесия ионного обмена различных пар ионов. Значения этих параметров существенно (в 2-4 раза) меньше, чем значения радиусов свободных гидратированных ионов в растворе, найденных различными экспериментальными методами. Вероятно, автор понимал, что при этом должна иметь место существенная дегидратация, которая не может быть «бесплатной»; учет хотя бы половин полных энергий гидратации катионов показал бы, что эти величины сопоставимы с энергиям кулоновского взаимодействия. Дж. Поли этого не учел.

2. Почему мы так высоко оцениваем модель Дж. Эйзенмана [5], хотя она является моделью для качественной харак- теристики рядов селективности? Не только из-за понимания, что с помощью простых моделей большего и не добиться. Высокая оценка связана с тем, что модель Эйзенмана не является подгоночной. Автор использует табличные значения кристаллохимических радиусов, пренебрегая возможностью существования молекул воды между фиксированным ионом и противоионом. Автор делает предположение, что остаются только гидратирующие молекулы воды, расположенные с периферийной части противоионов, взаимодействующих с ионитом, и использует табличные значения энергий дегидратации противоионов, уменьшая их ровно в два раза. Такая приближенная, но фундаментальная модель позволила Дж. Эйзенману предсказать, что, какие бы мы не взяли ионообменники, возможны всего 11 типов рядов селективности для однозарядных катионов, что и подтвердилось впоследствии многими экспериментальными исследованиями. Реагируя теперь на замечание В.А. Шапошника [3] по поводу формул, использованных Д. Райхенбергом [6], а именно, того, что в этих формулах отсутствуют «числа зарядов ионов, величина диэлектрической постоянной», укажем, что в соответствии с физическими предположениями, осознанно заложенными в них Дж. Эйзенманом, речь идет об однозарядных ионах и о кристаллохимических радиусах (без воды между ионами).

3. Почему в наших комментариях [2] были критические замечания в связи с тем, что В.А. Шапошник в своем подходе к теории селективности воспользовался моделью Дж. Поли (назовем ее так, хотя - это просто нормированная формула для разности энергий кулоновского взаимодействия) и только этой моделью? Из-за двух причин: а) нам кажется, что развивая какую-нибудь теорию или гипотезу, надо пользоваться не только первыми шагами предшественников, но и последующими или наиболее признаваемыми достижениями. 
Важны те результаты, которые развивают наиболее выдающиеся достижения, а не первые шаги; б) суть отказа В.А. Шапошника от энергий дегидратации, которую Владимир Алексеевич разъяснил в своих комментариях [3], можно изложить просто: раз теория гидратации развита недостаточно и раз нет табулированных, совпадающих друг с другом экспериментальных значений радиусов гидратированных ионов и, наконец, раз структура гидратных оболочек не простая (о чем также свидетельствуют квантово-механические расчеты), то нет и смысла учитывать энергии дегидратации. На наш взгляд, с этим принципиально невозможно согласиться. Вопервых, сегодняшнее состояние теории гидратации не такое плачевное. Вовторых, неучет энергий дегидратации приводит к еще большим ошибкам. Все это означает только, что простые модели, основанные на энергии кулоновского взаимодействия (с учетом или без учета энергий гидратации) могут быть только качественными. Хотя следует отметить, что такую модель до количественных оценок довел Д. Райхенберг [6], добавив в соответствующие соотношения термы для энергий близкодействующих взаимодействий, включая дисперсионные.

4. В чем состоит наше несогласие с использованием дробных зарядов? Повторим в точности то, что по этому поводу было сказано в комментариях [2], имея ввиду, что формула Кулона основана на модели разделенных точечных зарядов. «Если принимается модель точечных зарядов, то какая бы она ни была грубой, расчет надо вести по формуле Кулона. Если Вы учитываете делокализацию зарядов, то необходимо понимать, что придется описывать функции распределения потенциалов и зарядов и решать уравнение Пуассона. Нам кажется, что нельзя поправлять расчеты с помощью использования величин дробных зарядов в точечной модели, так как в ней изначально никакая делокализация не предусмотрена. Никакой степени обоб- щения с помощью таких моделей нельзя добиться. Это приводит к такому же результату, как и введение каких-то подгоночных эмпирических параметров, разных для разных случаев. Легче просто запомнить таблицы известных равновесных коэффициентов». Как видно, здесь ничего не говорится о молекулах органических или неорганических веществ с перекрывающимися атомными орбиталями и неразделенными зарядами, или, что тоже самое, об эффективных зарядах частей полярных молекул, часто используемых для характеризации таких молекул. Обо всем этом, как бы опровергая наши выводы, пишет в [3] сам автор. Мы к этому спору не имеем отношения, однозначно сформулировав раньше только то, что относится к кулоновским взаимодействиям, имея ввиду следующее: формула Кулона описывает взаимодействие полностью разделенных зарядов (ионов, элементарных частиц). Физика не позволяет нам использовать дробное значение разделенных зарядов, они могут быть только целочисленными и кратными заряду электрона. (Речь не идет о кварках, заряд которых дробный, но кварки, как правило, входят в состав частиц с целочисленными зарядами).

5. Можно ли применить уравнение Пуассона к ионообменникам? Разумеется, теоретическим обоснованием теории селективности Г. Грегора и Л. Лазара явилось решение уравнения Пуассона Больцмана (УПБ) для системы параллельных пластин с двойными электрическими слоями (ДЭС), что явилось хорошей моделью для сильнокислотных ионитов [7]. Позже В. Хёль довел этот подход до возможности расчета коэффициентов равновесия обмена ионов в многокомпонентных системах практически для всех типов ионитов [8,9]. Комментарии, приводимые в [3] по логике приближенного решения этого уравнения в теории Дебая-Хюккеля, вполне правильные. Но за прошедшие 100 лет и теория и возможности получения хорошо сходящихся численных решений продвину- 
лись далеко. Примеров работ в этом направлении великое множество. Но прежде всего, в качестве примеров, демонстрирующих возможности применения уравнения Пуассона с распределением Больцмана к концентрированным растворам электролитов, приведу хорошо знакомые мне работы $[10,11]$. Кроме того, есть возможность использовать промежуточные математические выражения разных решений уравнения Пуассона-Больцмана и проводить дальнейший анализ с использованием современных представлений в физической химии. Ближайшими примерами являются работы $[12,13]$, в которых с участием автора этих строк были решены весьма сложные задачи для сильноосновных ионитов, а также аэрогелей (гидрогелей) на основе графена для емкостной деионизации с использованием промежуточных выкладок одномерного решения УБП в теории диффузного ДЭС Гуи-Чепмена и решения этого же уравнения в сферических координатах в теории электролитов Дебая-Хюккеля. Подходы на основе уравнения Пуассона настолько сильно продвинулись, что развитая в последние десятилетия электродиффузионная теория - модель PNP (Пуассона-НернстаПланка) широко используется для решения динамических (макрокинетических) задач как в области ионного транспорта в клетках [14], так и для описания процессов переноса в концентрированных системах через ионообменные мембраны [15].

6. Какова взаимосвязь между селективностью в ионном обмене и водородной связью? Нам кажется, что однозначно на этот вопрос сейчас не может ответить никто, хотя в работах В. А. Шапошника и его коллег делается такая попытка, к чему следует относиться с большим уважением. Недопонимание, наверное, связано с тем, что трудно согласиться с главным утверждением, которое делается в [3] по этому поводу: «Так как энергия активации транспорта ионов в ионообменниках является суммой энергий водородных связей и электростатического взаимодействия, то фактически селективность определяется только кулоновскими силами». Кроме водородных связей и дальнедействующего электростатического взаимодействия есть еще ион-дипольные, дипольдипольные, а также дисперсионные взаимодействия (главным образом с участием молекул воды). Кроме этого в энергию активации необходимо включить трансляционную энергию (энергию переноса) заряженных частиц, даже после того как они «оторваны». Именно поэтому мы и написали в [2], что для проведения количественных оценок надо использовать не одно значение, а разность значений двух энергий активаций, где вторая энергия относится к переносу в растворах. Кроме того, из простых физических соображений следует, что в зависимости от числа молекул воды, внедренных между взаимодействующими ионами, теоретически может быть как ослабление взаимодействия так и усиление. (Увеличивается диэлектрическая постоянная - это ослабление. Увеличивается расстояние между зарядами - тоже ослабление. Уменьшается расстояние между поляризованными диполями и фиксированным ионом - это вклад в усиление). Чего тут больше - неясно, хотя нас учили и до сих пор учат студентов и аспирантов, что более сильные взаимодействия, как правило, связаны с дегидратацией.

В любом случае пожелаем успехов В.А. Шапошнику и его коллегам в их исследованиях.

\section{Список литературы}

1. Шапошник В.А. // Сорбичонные $u$ хроматографические прочессы. 2020. Т.20.

№ 1. C. $48-53$.

2. Хамизов Р.Х., Долгоносов А.М. // Сорб- 
иионные и хроматографические прочессы. 2020. T. 20. № 5. C. 641-646.

3. Шапошник В.А. // Сорбицонные и хроматографические проиессы. 2021. Т. 21. № 2. C. 285-290.

4. Pauley J.L. // J. Amer. Chem. Soc. 1954. Vol. 76. pp. 1422-1425.

5. Eisenman G., Rudin D.O., Casby J.U. // Science. 1957. Vol. 126. pp. 831-834.

6. Ионный обмен / ред. Я. Маринского. М. Изд-во Мир. 1968. 565 с.

7. Lazare L., Sundheim B.R., Gregor H.P. // J. Phys.Chem. 1956. Vol. 60. No 5. pp. 641-643.

8. Hoell W.H., Horst J., Eberle S.H. In. Ion Exchange and Solvent Extraction, a Series of Advances. Vol. 11. (Eds. J.Marinsky, Y.Marcus). N.-Y., Basel. Hong Kong: Macel Dekker. 1992. pp. 151-206.
9. Horst J., Hoell W.H., Eberle S.H. // React. Polym. 1990. Vol. 13. pp. 209-231.

10.Тихонов Н.А. // Доклады АН. 2010. T. 434. № 2. C. 175-177.

11. Tikhonov N.A., Sidelnikov G.B. // J. Math. Chemistry. 2013. Vol. 51. pp. 27462756.

12.Tikhonov N.A., Tokmachev M.G., Bakhia T., Khamizov R.Kh. // J. Math. Chemistry. 2021. Vol. 59. pp. 1054-1067.

13. Sidelnikov G.B., Tikhonov N.A., Khamizov R.Kh., Krachak A.N. // Math. models comp. simulat. 2013. Vol. 5. No 6. pp. 501-510.

14.Miękisz J., Gomułkiewicz J., Miękisz S. // Math. Applicanda. 2014. Vol. 42. No 1. pp. 39-62.

15.Коваленко А.В. // Фундамент. Исследования. 2015. № 11. С. 59-65.

\title{
Continued discussion of the article by V.A. Shaposhnik "Electrostatic theory of ion exchanger selectivity"
}

\author{
(C) 2021 Khamizov R.Kh.
}

\section{Institute of Geochemistry and Analytical Chemistry of Russian Academy of Sciences, Moscow}

The discussion paper by R. Kh. Khamizov is devoted to the materials presented by Professor V.A. Shaposhnik in the journal "Sorption and Chromatographic Processes" (2021, V.21, No 1). The article by R.Kh. Khamizov and A.M. Dolgonosov "Focus on the article by V.A. Shaposhnik "Electrostatic Theory of Selective Ion Exchangers"' considers approaches to the issue, including the one presented in the model by J. Pauley used to determine the equilibrium constants of ion exchange.

The model of G. Eisenman appears to be a more correct selectivity model, even though it only acts as a qualitative characteristic. The appeal of the Eisenman's model stems from the fact that it has not been adjusted. For his calculations Eisenman used table values of crystal-chemical radii assuming that there are no water molecules between a fixed ion and a counterion.

Critical remarks about V. A. Shaposhnik's approach to the theory of selectivity were made in connection with the fact that when a hypothesis is developed, it is necessary to use not only the first steps of your predecessors, but also their subsequent (or most recognised) achievements. Therefore, a disagreement was expressed with the position of V. A. Shaposhnik who chose to ignore the dehydration energy. A failure to take into account the energy of dehydration when considering the selectivity of ion exchangers leads to very serious errors. It should be noted that D. Reichenberg successfully used the qualitative model based on the energy of the Coulomb interaction to generate quantitative estimates by introducing the therms for the energies of short-range interactions, including dispersion interactions into the corresponding relations.

The author of this article believes that it is impractical to correct the calculations using fractional charges in the Coulomb model (which does not provide for delocalisation). Considering the applicability of Poisson's equation for ion exchangers, the author of this article recommends reading his works (in collaboration with N. A. Tikhonov), in which the authors solved very complex problems for strongly basic anionexchangers and graphene-based hydrogels.

Keywords: ion exchange, selectivity models, Coulomb interaction, hydration energy, hydrogen bond.

\section{References}

1. SHaposhnik V.A., Sorbtsionnye i khromatograficheskie protsessy, 2020, Vol. 20, No 1, pp. $48-53$.
2. Khamizov R.H., Dolgonosov A.M., Sorbtsionnye i khromatograficheskie protsessy, 2020, Vol. 20, No 5, pp. 641-646.

3. Shaposhnik V.A., Sorbtsionnye i khromatograficheskie protsessy, 2021, Vol. 21, No 2, pp. 285-290. 
4. Pauley J.L., J. Amer. Chem. Soc., 1954, Vol. 76, pp. 1422-1425.

5. Eisenman G., Rudin D.O., Casby J.U., Science, 1957, Vol. 126, pp. 831-834.

6. Ionnyj obmen, red. YA. Marinskogo, M., Izd-vo Mir, 1968, 565 p.

7. Lazare L., Sundheim B.R., Gregor H.P., J. Phys.Chem., 1956, Vol. 60, No 5, pp. 641-643.

8. Hoell W.H., Horst J., Eberle S.H., In. Ion Exchange and Solvent Extraction, a Series of Advances, Vol. 11, (Eds. J.Marinsky, Y.Marcus), N.-Y., Basel. Hong Kong: Macel Dekker, 1992, pp. 151-206.

9. Horst J., Hoell W.H., Eberle S.H., React. Polym., 1990, Vol. 13, pp. 209-231.

Хамизов Руслан Хажсетович - д.Х.н., заведующий лабораторией сорбционных методов, ГЕОХИ РАН, Москва
10.Tikhonov N.A., Doklady AN, 2010, Vol. 434, No 2, pp. 175-177.

11. Tikhonov N.A., Sidelnikov G.B., J. Math. Chemistry, 2013, Vol. 51, pp. 2746-2756.

12.Tikhonov N.A., Tokmachev M.G., Bakhia T., Khamizov R.Kh., J. Math. Chemistry, 2021, Vol. 59, pp. 1054-1067.

13. Sidelnikov G.B., Tikhonov N.A., Khamizov R.Kh., Krachak A.N., Math. models comp. simulat, 2013, Vol. 5, No 6, pp. 501-510.

14.Miękisz J., Gomułkiewicz J., Miękisz S., Math. Applicanda, 2014, Vol. 42, No 1, pp. 39-62.

15.Kovalenko A.V., Fundament. Issledovaniya, 2015, No 11, pp. 59-65.

Khamizov Ruslan Kh. - Dr. Sci. (Chem) Head of Laboratory of Sorption Methods, GEOKHI RAS, Moscow, e-mail:khamiz@mail.ru 